Conclusion: Treatment with diclofenac sodium and ketoprofen can affect the balance of XO/XDG activity and increase the antioxidant potential of the blood.

Reference: 1. Zborovskaya IA et al. Russ J Pain 2018;3:47

\section{P715 \\ MONOCYTE EXTRACELLULAR TRAPS IN RHEUMATOID ARTHRITIS}

M. A. Mamus ${ }^{1}$, E. A. Tikhomirova ${ }^{1}$, E. E. Mozgovaya ${ }^{1}$, A. S. Trofimenko², S. A. Bedina', S. S. Spitsina², I. A. Zborovskaya $^{2}$

${ }^{1}$ Research Institute of Clinical and Experimental Rheumatology A.B. Zborovsky, ${ }^{2}$ Research Institute of Clinical and Experimental Rheumatology A.B. Zborovsky, Volgograd State Medical University, Volgograd, Russia

Objective: To study the propensity of circulating monocytes for spontaneous and induced formation of extracellular traps in rheumatoid arthritis (RA).

Methods: The study was performed according to bioethical standards. 15 patients over 18 years old with verified RA, disease history less than 2 years, and DAS28 score not exceeded 2.6 were included in the study. The 2010 ACR/EULAR criteria were used for verification. 9 patients $(60 \%)$ were positive for anti-citrullinated protein antibodies (ACPA). 15 healthy volunteers were included in the control group. RA patients and healthy volunteers were comparable by sex and age. Circulating monocytes were isolated using density gradient centrifugation of buffy coat $(700 \mathrm{~g}, 15$ min) over slightly hyperosmolar ficoll-amidotrizoate gradient with density $1068 \mathrm{~kg} / \mathrm{m}^{3}$. We induced the extracellular traps formation using $100 \mathrm{nM}$ PMA in PBS. The extent of extracellular traps formation by monocytes was assessed using fluorescent microscopy. Results are expressed as percent of netting cells in the specimen. Central tendencies and variabilities are expressed as Mean $(95 \% \mathrm{Cl})$.

Results: Mean age of patients was $56.2 \mathrm{y}$, mean disease duration was $1.4 \mathrm{y}$. The average proportion of monocytes with spontaneous trap formation was significantly higher in RA patients $8.4 \%$ (6.6$12.0)$ comparing with the control group $5.8 \%(3.8-7.0)(p<0.05)$. The frequency of spontaneous trap formation by monocytes in ACPA-positive RA patients with RA is similar to their negative counterparts. The average proportion of monocytes with induced trap formation was significantly higher in RA patients $27 \%$ (20.1-33.2) comparing with the control group 17.6\% (15.321.7) $(p<0.05)$. Significant morphological differences between monocyte extracellular traps in RA patients and control group are not revealed.

Conclusion: RA is a systemic inflammatory autoimmune disorder. The pathogenesis of RA is complex. The formation of extracellular traps by the monocytes/macrophages may be one of the links in the pathogenesis of RA.
P716

DATA ANALYSIS OF THE "REGISTRO ESPAÑOL DE FRACTURAS" FLS SEIOMM (REFRA)

M. J. Moro-Alvarez ${ }^{1}$, J. M. Cancio ${ }^{2}$, J. Mora ${ }^{3}$, E. Duaso ${ }^{4}$, M. J. Montoya ${ }^{5}$, G. Refra ${ }^{6}$

${ }^{1}$ Infanta Leonor University Hospital, Madrid, ${ }^{2}$ Geriatrics Service. Centre Sociosanitari El Carme., Badalona Serveis Assistencials (BSA), ${ }^{3}$ Geriatrics Service San Carlos Clinical Hospital, Madrid, ${ }^{4}$ Igualada Hospital, Barcelona, ${ }^{5}$ Virgen Macarena University Hospital, Sevilla, ${ }^{6}$ SEIOMM, Madrid, Spain

Bone fragility fractures represents a major public health issue that, currently, does not have the adequate procedures of prevention, diagnosis, assessment, intervention and monitorization of the patients. The mortality associated to fragility fracture in the EU countries is higher than in many other chronic diseases. In Spain in 2017 there were around 330000 fragility fractures, with a sanitary cost of 4200 million euros. The ongoing and prospective Registro Español de FRActuras (REFRA-FLS SEIOMM) started in 2018. The aim was to create a multicentric register of the epidemiologic, clinic, functional and healthcare features of the fragility fracture patients, as well as their monitorization. The aim of this communication is to get to know the first preliminary descriptive data.

Attended people with fragility fracture diagnosis in some of the REFRA participant hospitals between July 2018 and December 2019 , was included. In the statistical analysis was used the mean and the standard deviation or the mean and the interquartile ranks for the numeric variables; and the percentage for the categorical variables.

It was registered 1556 bone fragility fracture patients; with a mean age of $79,80 \%$ of women and $20 \%$ of men. Only $25.89 \%$ had the osteoporosis diagnostic, and only $12.08 \%$ osteoporosis treatment; although $32.78 \%$ of the patients had a previous fracture, with the prevalence of radius distal and vertebral. Fracture index recollected is $59.14 \%$ hip, $10.30 \%$ radius distal, $12,74 \%$ vertebral, $7.91 \%$ humerus, $3.39 \%$ pelvic, $6.25 \%$ others. The average time to fracture capture is $140.9 \mathrm{~d}$. Cardiovascular and endocrine diseases are the most frequent comorbidities associated. The $60.78 \%$ of the patients have a high fall risk and fracture risk, through FRAX, for fracture higher than $16.16 \%$ and for hip fracture of $9.08 \%$. 